\title{
Genome-wide functional analysis of SSR for an edible mushroom Pleurotus ostreatus
}

\author{
Jibin $\mathrm{Qu}^{1,2}$, Chenyang Huang ${ }^{1,2}$, Jinxia Zhang ${ }^{1,2, *}$ \\ ${ }^{1}$ Institute of Agricultural Resources and Regional Planning, Chinese \\ Academy of Agricultural Sciences, Beijing, China \\ ${ }^{2}$ Key Laboratory of Microbial Resources, Ministry of Agriculture, \\ Beijing, China \\ * Corresponding author
}

Address: 12 Zhongguancun South Street, Beijing 100081, China

Tel: $+86-10-82108692$

Email: zhangjinxia@caas.cn 


\section{Abstract}

1 Simple sequence repeats (SSRs) play specific roles in many biological activities. In

2 this paper, we focused on SSRs in the genome of Pleurotus ostreatus, which is a

3 widely cultivated edible mushroom. The distribution curves of SSRs and exons are

4 opposite throughout the genome, which means that SSRs are mostly located in

5 non-coding regions. A comparative analysis of nine fungi suggests that

6 Agaricomycotina fungi have similar SSR distributions. Functional enrichment

7 analysis on the SSR-containing gene set uncovers enriched functions about

8 environmental interactions and important cellular functions for life. Trinucleotide

9 SSRs account for an extremely high fraction of all SSRs, and in exonic regions, they

10 are equivalent to inserting repeating amino acids (RAA) into the protein sequences.

11 The RAA indel could partly explain some enriched functions of the genes they

12 modify. Agaricomycotina fungi have similar distributions of RAAs, indicating that

13 this may be a potential common mechanism for some specific functions.

14 Keywords: simple sequence repeat; Pleurotus ostreatus genome; functional 15 enrichment analysis; repeating amino acid. 


\section{Introduction}

Simple sequence repeats (SSRs), also known as microsatellites or short tandem repeats (STRs), are 1-6 repeating base pairs of DNA[1-3]. SSRs have been detected ubiquitously in the genomes of every prokaryotic and eukaryotic organism[4-8]. Such repetitions occur primarily due to slipped-strand mispairing and subsequent errors during DNA replication, repair, or recombination[9]. These SSR regions, which are spread extensively throughout genomes and can encompass tens to hundreds of base pairs, are typically codominant and have a high mutation rate[8]. The SSRs result in a high frequency of polymorphisms between alleles, especially for long repetitive loci[9]. SSRs are therefore an ideal marker for genetic analyses[11-13]. SSRs also play important roles in gene regulation, evolution, chromatin organization, DNA replication, and the cell cycle[8,10,11].

Despite their widespread applications, little is known about the functions of SSRs in fungi[1,3]. In fact, there are only a limited number of studies on this particular type of sequence in fungal species[1] with the exception of Saccharomyces cerevisiae $[12,13]$. With the development of next-generation sequencing technology, many fungal genomes have been sequenced[14], but detailing the characteristics of massive genomes has not attracted enough attention.

In this paper, we focus on an edible fungus Pleurotus ostreatus, which occupies the second most important position in the world mushroom market and is the most popular mushroom in China. This fungus can grow easily in various temperature ranges on a variety of organic substrates, including agricultural waste. $P$. ostreatus is 
40

41

42 and released[18,20].

43 Before the publication of the P. ostreatus genome, only a few SSRs had been 44 set.

widely cultivated[15] and represents an important complementary foodstuff. In addition to its delicious taste and nutritional value, this mushroom also has health-promoting effects[16,17]. The complete genome of $P$. ostreatus was sequenced discovered to serve as biomarkers for genetic research [19]. We detected all of the SSRs in the PC15 genome, providing thousands of new candidate markers. Next, we observed some general characteristics of the SSRs in this organism and noticed a novel frequency of trinucleotide SSRs. We then performed functional enrichment analyses to determine the biological functions of these SSR regions. Lastly, we discussed the properties of repeating amino acids encoded by trinucleotide SSRs in exonic regions and linked these properties to the functions of the SSR-containing gene 


\section{Materials and Methods}

\subsection{The PC15 genome}

The PC15 genome was published in 2010[18] and released recently[20], and is available via the JGI Genome Portal MycoCosm (http://jgi.doe.gov/fungi) and GeneBank (accession number AYUK00000000). It was assembled into twelve scaffolds, which represented general chromosome structures. This genome is 34.3 Mbp and eight scaffolds represent complete chromosomes (there are telomeres on both ends), three scaffolds are parts of chromosomes (telomere is found just on one end), and one scaffold is a sequence segment. Exonic regions were highlighted and some basic annotations (GO, KEGG, Interpro, KOG and Signalp) were made.

\subsection{Detecting perfect SSRs in the genome}

In this paper, only perfect SSRs whose repeats were completely identical were considered, because the patterns of imperfect SSRs are too complex and impossible to define. There are six SSR patterns for repeating units of 1-6 base pairs, named mononucleotide, dinucleotide, trinucleotide, tetranucleotide, pentanucleotide, and hexanucleotide, respectively. The accepted lower bounds of SSR length were as follows: 12nt for mononucleotide, dinucleotide and trinucleotide SSRs; 16nt for tetranucleotide SSRs; 20nt for pentanucleotide SSRs; and 24nt for hexanucleotide SSRs[2]. Our programs were executed in the Matlab environment (for the source code, see the supplement).

\subsection{The SSR patterns in detail}

When considering the SSR pattern in detail, we identified patterns of SSRs that 
appeared more frequently. Here, repeating sequences were considered to be equivalent if they were on the complement strand and in a different reading frame. For example, $(\mathrm{AT})_{\mathrm{n}}$ is equivalent to $(\mathrm{TA})_{\mathrm{n}}$, and $(\mathrm{ATG})_{\mathrm{n}}$ is equivalent to $(\mathrm{TGA})_{\mathrm{n}}$, $(\mathrm{GAT})_{\mathrm{n}},(\mathrm{ATC})_{\mathrm{n}},(\mathrm{TCA})_{\mathrm{n}}$ and $(\mathrm{CAT})_{\mathrm{n}}$.

\subsection{Determining the SSR-containing gene set and the locations of SSRs}

In addition to being used as genetic markers, SSRs have special functions in gene regulation, evolution, chromatin organization, DNA replication and many other biological activities[21]. It is therefore important to investigate the functions of SSR loci.

The mutability of SSRs suggests that their functions are not likely to be conserved, for example functions related to environmental or other responses to stimuli. Our research focused on the genes whose sequences contained SSRs. Because the PC15 genome just defined exonic regions, we simplified the entire sequence of a gene to be the region from the stop codon of the upstream gene to the stop codon of this gene. In addition to exons, the whole considered sequence included introns and intergenic regions. The SSR-containing gene set was thus defined by SSRs that overlapped this considered region.

It was apparent that some SSR regions might bridge adjacent exons and non-coding regions. We defined the location of a SSR by the midpoint of the repeated segment.

\subsection{Defining regions with abundant SSRs}

In some regions of the genome, SSRs appeared more frequently and these segments represented a larger proportion of the sequence. This meant that these regions had a 
higher mutation rate. The ordering of genes in eukaryotic genomes is not completely random. The chromosome structure determines cofunctionality among adjacent genes and this may be influenced by cis-regulatory elements, bidirectional transcription, gene interactions, etc.[22]. We focused on the sets of genes in SSR-abundant regions. We divided the genome into consecutive segments with equivalent length $P(\mathrm{bp})$ and counted the length of SSRs in every segment. Segments with SSR length larger than $R$ (bp) were defined as SSR-abundant regions and genes in these regions were our candidates for analysis. Here we used $P=5000$ and $R=100$, thus the SSR length of these regions accounted for at least $2 \%$ of the $5000 \mathrm{bp}$ segment; this proportion was twenty-times the average SSR length of the genome.

\subsection{Significant functions enriched in the gene set containing SSRs}

Gene Ontology (GO) annotations contain functional information about a gene product[23]. The PC15 genome was annotated simply and the GO functions of many genes were made available. From a systems biology standpoint, it is not sufficient to explain a complex biological problem by considering only one or a few genes because a large number of genes control a biological system. One gene may have many functions whereas many genes may possess one function. We therefore did not assess the functions of single genes containing SSRs but rather the enriched functions of gene sets containing SSRs. To detect significant enriched functions of the sets of genes containing SSRs, a statistical analysis was applied to this information about gene function. A commonly used statistic compares GO term enrichments in a gene set of interest to a reference gene set. We used the Blast2GO software to perform the 
119 functional enrichment analysis. Blast2GO computes a Fisher's Exact Test and applies 120 a robust FDR (false discovery rate) correction for multiple testing and returns a list of 121 significant GO terms ranked by their corrected or one-test P-values[24,25]. All the 122 genes were used as the reference set of enrichment analysis, and the genes with SSRs 123 were used as the test set. 


\section{Results and Discussion}

\subsection{Overview of SSRs in the genome}

We identified 2114 perfect SSRs in the PC15 genome (see supplement table 1). The average frequency of SSRs in the genome was approximately 62 SSRs/Mbp (mega base pairs) and the average length was $1025 \mathrm{bp} / \mathrm{Mbp}$, accounting for $0.1 \%$ of the genome. The SSR densities of other fungi range from tens to hundreds of $\mathrm{SSR} / \mathrm{Mbp}[1,3]$, so our result was within this range. Figure 1a shows a comparison of SSRs patterns among nine fungi: Saccharomyces cerevisiae [26], Schizosaccharomyces pombe [27], Magnaporthe grisea [28], Schizophyllum commune [29], Coprinopsis cinerea [30], Laccaria bicolor [31], Agaricus bisporus [32], Pleurotus ostreatus [20], and Ustilago maydis. Each of these fungi exhibited a relative lack of tetranucleotide, pentanucleotide and hexanucleotide SSRs. An impressive phenomenon for Agaricomycotina fungi was that the frequency of trinucleotide SSRs was larger than that of other SSRs. A basic hierarchical clustering was performed to partition the different fungi based on the SSR density of different repeat units. In the hierarchical clustering process we selected the Euclidean distance of SSR densities as the dissimilarity between organisms, and the shortest distance as the distance between clusters. The five Agaricomycotina fungi were clustered together (figure 1b). This suggests that Agaricomycotina fungi had a similar SSR density and Ustilago maydis was more similar with the Agaricomycotina fungi for SSR density than others because they belong to Basidiomycota fungi. S. commune was the nearest species to $P$. ostreatus by their SSR distribution. These results met the 
common taxonomy of these species basically [33].

The SSRs were distributed non-uniformly in different parts of the genome sequence.

Some segments were rich in SSRs whereas others lacked them. Figure 2 shows the varying density of SSRs and the distribution of exons in the whole genome. The distributions of SSRs and exons are complement, which means that most SSRs are located in non-coding regions. The Pearson correlation coefficient between length of SSR and length of exon per unit length $(50,000 \mathrm{bp})$ is -0.29 , with the p-value $4.98 \times$ $10^{-15}$

There are six types of SSRs with different lengths of the repeat unit. The distributions of the density of SSRs in different scaffolds are shown in figure 3a. It was clear that trinucleotide SSRs were the most frequent type of SSRs. The correlation coefficient between scaffold length and the number of SSRs was 0.96, showing an obvious linear relationship.

Our study also observed the SSR patterns in details (see supplement figure S1). An overrepresentation of $\mathrm{A} / \mathrm{T}$ repeats were compared to $\mathrm{C} / \mathrm{G}$ repeats, which has been observed in most organisms[3,9]. The distribution pattern of dinucleotide SSRs conformed to the general observations in fungi and some other organisms: AT and AG repeats outnumbered other repeats and CG repeats appeared rarely[1,3]. Common trinucleotide SSRs were $(\mathrm{ACG})_{\mathrm{n}}$ and $(\mathrm{GAG})_{\mathrm{n}} \cdot(\mathrm{CCTC})_{\mathrm{n}},(\mathrm{CCAT})_{\mathrm{n}},(\mathrm{GCGT})_{\mathrm{n}}$ appeared more frequently in tetranucleotide SSRs whereas others appeared rarely. (TGAAC) was the most common pentanucleotide SSR, and (CCCTAA) n $_{n}$ was the most common hexanucleotide SSRs because it was the marker of telomere. 

be translated to amino acids from mRNAs, which are transcribed from exonic regions of DNA. So, in exonic regions, trinucleotide and hexanucleotide SSRs are important,

172 but other SSRs may be meaningless. We wanted to determine how many SSRs were located in exonic and non-coding (intronic or intergenic) regions of the genome. The SSR distributions in exonic and other regions are shown in figure $3 \mathrm{~b}$. The frequency

175 with which trinucleotide and hexanucleotide SSRs were located in exonic and other regions was significantly larger than other SSRs (t-test p-values of $2.4 \times 10^{-7}$ and 3.9

$177 \times 10^{-6}$ for trinucleotide and hexanucleotide SSRs, respectively), and other SSRs rarely appeared in exonic regions and occurred mostly in non-coding regions. It is reasonable that indels of the trinucleotide and hexanucleotide SSRs in exons may affect the protein structure by inserting amino acids without affecting the surrounding sequence. However, insertions of other SSRs into exonic regions may influence the translation of mRNA because of SSR polymorphisms within filial generations (this frequent terms. Catalytic Activity (27.9\%) was highly represented in the Molecular case appeared rarely because it compromises the robustness of biological systems).

\subsection{Functional enrichment analysis for the subset of genes containing SSRs}

There were 1497 genes whose sequence contained SSRs, which were used as the test set of the enrichment analysis. GO terms were available for approximately $44.8 \%$ (671) of the genes and these ontologies are shown in supplement figure S2[34]. In the Cellular Component ontology, Cell (17.1\%) and Cell Part (17.1\%) were the two most Function ontology, followed by Binding (24.7\%). In the Biological Process ontology, 
191

192

193

Metabolic Process (20.1\%) and Cellular Process (17.8\%) were the top two GO terms. Using the Blast2GO software (FDR cut-off of 0.05 ), we revealed the significant functions of these genes (figure 4 and supplement table 2). Ten GO functions were over-represented in the SSR gene set, while one function was under-represented in the SSR gene set. To detect the specific functions of the SSR gene set, we considered more GO functions (with less significance) by increasing the p-value cutoff to 0.005 , and got $67 \mathrm{GO}$ functions of significant enrichment (see the supplement table 2).

Several aspects of the functions provided in the supplement table 2 can be substantiated. First, SSRs act as 'tuning knobs' to provide an evolutionary advantage allowing for a fast adaptation to new environments[35]; our result contained many associations with environmental interactions, for example signal processing (e.g., GO: 0023051, regulation of signaling; GO:0046578, regulation of Ras protein signal transduction), membrane related activities (e.g., GO: 0015462 , protein-transmembrane transporting ATPase activity) and epigenetic modification (e.g., GO: 0004402, histone acetyltransferase activity; GO: 0071103, DNA conformation change). $P$. ostreatus can be cultivated on a wide variety of substrates that contain lignin and cellulose without the requirement of costly processing methods or the addition of enrichment materials. Furthermore, they demand few environmental controls and diseases and pests rarely afflict their fruiting bodies [36,37]. They can therefore be more simply and inexpensively cultivated because they are more tolerant of different environments than other edible mushrooms. SSRs may have important effects on the molecular mechanisms of environmental adaptation. 
214 SSRs were over-enriched for the nucleus compartment ( $\mathrm{p}$-value is $3.52 \times 10^{-5}$ ) but 215 under-enriched for mitochondrion ( $\mathrm{p}$-value is $6.2 \times 10^{-4}$ ) and cytoplasm ( $\mathrm{p}$-value is $2.39 \times 10^{-3}$ ), over-enriched for the regulation of nitrogen compound metabolic processes ( $\mathrm{p}$-value is $2.4 \times 10^{-3}$ ) but under-enriched for cellular nitrogen compound biosynthetic process ( $\mathrm{p}$-value is $1.00 \times 10^{-2}$ ), over-enriched for hydrolase activity 219 (p-value is $3.26 \times 10^{-4}$ ) but under-enriched for oxidoreductase activity ( $\mathrm{p}$-value is 2.00 220 $\left.\times 10^{-6}\right)$ genes containing SSR. For fungi, this function is more concerned for biodegradation, which is pivotal to the earth's carbon cycle [38]. For example, manganese-peroxidase is the most common enzyme to degrade lignin [38-40]. In PC15 genome, 9 genes were detected to encode manganese-peroxidase (mnpl-mnp9) [40], just one of them (mnp3) had the structure of SSR. Phenol oxidase (laccase) is another kind of enzyme especially for fungi to degrade lignin [41]. For 12 genes encoding laccase (lacc1-lacc12) in PC15 genome, just one of them (lacc1) contained the SSR segment. There were 120 genes expressed, which were grouped to seven functional annotations.

231 Four of the expressed genes contained the SSR segment. In the nucleic acid metabolism group, there were 2 genes containing SSR (geneID: 1112676, 1090372), they appeared in the functions of nucleic acid binding and helicase activity in our GO enrichment results which was closely related to nucleic acid metabolism. In the 
cellular signalling group, there was one gene containing SSR (geneID: 1093414), with the function enzyme regulator activity. And in the group of hypothetical protein, one gene containing SSR (geneID: 1101746) appeared in our result with multiple functions about binding, regulation and transcription.

By our method, we also derived a smaller gene set including 28 genes from the SSR-abundant regions. Some significant functions $(\mathrm{P}-\mathrm{value}<0.01)$ were obtained (table 1) through a functional enrichment analysis of this gene set. These functions mainly regarded the environmental defence response and ligase activity of glutamic. These regions contained the most abundant SSRs, so it is possible that these functions are associated with SSR polymorphisms.

\subsection{The functions of trinucleotide SSRs in exons}

In contrast to other SSR types in the P. ostreatus genome, there was a high frequency of trinucleotide SSRs. A trinucleotide motif in exons can be translated to specific amino acids, of which proteins are comprised, so these SSRs may affect the protein structure or play a role in gene regulation and transcription[35]. We identified all the trinucleotide SSRs that encode amino acids in exons. Table 2 details the repeating amino acids translated by trinucleotide repeats. It is clear that the more frequent codons resulting from trinucleotide repeats encode either small (Ser, Ala, Gly, Pro, Thr, Val) or hydrophilic amino acids (Arg, Glu, Asp, Lys). It has previously been suggested that codons encoding small/hydrophilic amino acids are better tolerated [21], and these trinucleotide motifs in coding regions likely confer a survival value to remain in a population[9]. 
As we described above, trinucleotide SSRs in exons may affect protein structure,

which is related to protein functions. We found similar functions between frequent trinucleotide SSRs and the set of genes containing SSRs.

Acidic and polar amino acid repeats (Asp and Glu) are significantly associated with transcription factors and protein kinases[43]. Repeats encoding these two amino acids were abundant and many functions in supplement table 2 were associated with TFs and protein kinases, for example nucleic-acid-binding transcription factor activity (GO: 0001071), small GTPase regulator activity (GO: 0005083), and regulation of Ras protein signal transduction (GO: 0046578). Glu participates in nitrogen metabolism and disposes of excess or waste nitrogen[44]. This is relevant for $P$. ostreatus because of the abundant repeat-encoded Glu and some uncovered functions regarding nitrogen metabolism (GO: 0051171, regulation of nitrogen compound metabolic process).

The residues of some amino acids are susceptible to numerous posttranslational modifications[36], for example Thr and Lys which had a higher proportions in our result. Inserting these amino acids might increase the possibility of modifications, such as lysine N-acetyltransferase activity (GO:0004468) and nucleoside-triphosphatase regulator activity (GO:0060589).

Repeats encoding Ser had been previously reported to be associated with membrane transporter proteins[43], and in our enriched functions of SSR-containing genes, there were also significant associations with transmembrane transporter activity (GO: 0008320, protein transmembrane transporter activity). 
As an edible fungus growing on plant materials and agricultural wastes, $P$. ostreatus had distinct functions about degradation, mating, nutrition productions and so on. In fact some Agaricomycotina fungi also shared these functions. Because repeating amino acids (RAAs) might affect the gene functions, comparative analyses of the Agaricomycotina fungi Schizophyllum commune, Coprinopsis cinerea, Laccaria bicolor, Agaricus bisporus, the Basidiomycetes Ustilago maydis and the Saccharomyces cerevisiae provided considerable insight into the evolution of the RAAs in fungi. Here the relative proportion of each RAA was considered because of the different genome and exome scale.

Figure $5(a, b)$ is the heat map of the proportions of RAAs for the seven organisms and the hierarchical clustering result for this matrix. Here we used the Euclidean distance of SSR densities as the dissimilarity between organisms, and the shortest distance as the distance between clusters. The proportions of RAAs are more similar between Agaricomycotina fungi, and U. maydis is nearer to Agaricomycotina fungi than $S$. cerevisiae because they belong to Basidiomycetes. It was explained that the evolution of RAA agrees with the taxonomy of fungi [35].

Some RAAs (Val, Ser, His, Tyr and so on) had stable proportions while some (Ala, Gln, Arg) varied strongly (see Figure 5(c)). Not all the RAAs were conserved in evolution. We could rank the level of conservation of different RAAs by the range of variety. 


\section{Conclusions}

Pleurotus ostreatus was the first completely sequenced edible fungus [14,18], and the most popular edible mushroom in China. This study links for the first time the physiological habits of $P$. ostreatus to its genomic sequence characteristics. Analysing SSRs in its genome is important to explain the mechanism of some physiological properties and is helpful for functional genomic research. This study also provides many SSR biomarker candidates for genetic research.

The distribution of SSRs was not uniform through the P. ostreatus genome. Trinucleotide SSRs appeared more frequently than others, and more than half of them were located in exons. Those SSRs not divisible by three were mostly found in non-coding regions. This distribution has been often observed for other Agaricomycotina species.

In our opinion, SSRs regulate biological activities mainly in two ways. The first is the modification of protein structure through the insertion of amino acids. These amino acids have certain functions themselves and can bind some ions, groups and motifs[45,46], or they can affect the protein activity by modifying its structure[47]. Above we have illustrated many functions of amino acid repeats. The second way to regulate biological activity is by modifying the sequences and affecting transcriptional regulation. It has been reported that SSRs can affect gene expression, mRNA splicing, transcription slippage, TF binding and other transcriptional activities through insertion into introns, UTRs or other non-coding regions[21]. The significant ontologies enriched in the SSR-containing gene set include many aspects of binding, 
transcription and regulation.

$P$. ostreatus can be widely cultivated, and it can adapt to different temperatures, humidity and substrates. It exists on every continent except Antarctica and grows throughout the year. One important application of SSRs is to distinguish different strains of the same species based on polymorphisms and high mutability because strains in different environments may have different SSR regions. This may be one of the mechanisms explaining the wide adaptivity of this species to new environments. We propose two lines of evidence. First, there was an extremely high proportion of trinucleotide SSRs and the chemical properties of these amino acids may facilitate environmental interactions. Second, the genes in which the SSRs were located were enriched for many GO functions regarding environmental interactions.

There are tight relationships for SSR density and repeating amino acids between Agaricomycotina fungi. These fungi have some specific functions, and the similar SSR distribution may be a potential common mechanism for these functions.

\section{Acknowledgements}

The sequence data were produced by the US Department of Energy Joint Genome Institute http://www.jgi.doe.gov/ in collaboration with the user community. This work was supported by National Basic Research Program of China (2014CB138303) and National Nonprofit Institute Research Grant of IARRP-CAAS (302-3). We would like to thank Prof. M. S. Goettel and Dr. Xiangli Wu for valuable suggestions about the manuscript. 


\section{References}

345 [1] J. Labbé, C. Murat, E. Morin, F. Le-Tacon, F. Martin, Survey and analysis of simple sequence

346 repeats in the Laccaria bicolor genome, with development of microsatellite markers. Curr Genet (2011) 57: 75-88.

[2] A. Merkel, N.J. Gemmell, Detecting microsatellites in genome data: variance in definitions and bioinformatic approaches cause systematic bias. Evol Bioinform Online (2008) 4: 1-6.

[3] H. Karaoglu, C.M.Y. Lee, W. Meyer, Survey of simple sequence repeats in completed fungal genomes. Mol Biol Evol (2005) 22: 639-649.

[6] S. Temnykh, G. DeClerck, A. Lukashova, L. Lipovich, S. Cartinhour, S. McCouch, Computational and experimental analysis of microsatellites in rice (Oryza sativa L.): frequency, length variation, transposon associations, and genetic marker potential. Genome Res (2001) 11: 1441-1452.

[7] J. Yonemaru, T. Ando, T. Mizubayashi, S. Kasuga, T. Matsumoto, M.Yano, Development of genome-wide simple sequence repeat markers using whole-genome shotgun sequences of sorghum (Sorghum bicolor (L.) moench). DNA Res (2009) 16: 187-193.

[8] P. Cavagnaro, D. Senalik, L. Yang, P. Simon, T. Harkins, C. Kodira, et al., Genome-wide 
[9] M.V. Katti, P.K. Ranjekar, V.S. Gupta, Differential distribution of simple sequence repeats in eukaryotic genome sequences. Mol Biol Evol (2001) 18: 1161-1167.

[10] T.P. Michael, S. Park, T.S. Kim, J. Booth, A. Byer, Q. Sun, et al., Simple sequence repeats provide a substrate for phenotypic variation in the Neurospora crassa circadian clock. PLoS One (2007) 2: e795.

[11] Y.C. Li, A.B. Korol, T. Fahima, A. Beiles, E. Nevo, Microsatellites: genomic distribution, putative functions and mutational mechanisms: a review. Mol Ecol (2008) 11: 2453-2465.

[12] D. Field, C. Wills, Abundant microsatellite polymorphism in Saccharomyces cerevisiae, and the different distributions of microsatellites in eight prokaryotes and S. cerevisiae, result from strong mutation pressures and a variety of selective forces. Proc Natl Acad Sci U S A (1998) 95: 1647-1652.

[13] K.D. Richards, M.R. Goddard, R.C. Gardner, A database of microsatellite genotypes for Saccharomyces cerevisiae. Antonie van Leeuwenhoek (2009) 96: 355-359.

[14] E. Morin, A. Kohler, A.R. Baker, M. Foulongne-Oriol, V. Lombard, L.G. Nagy, et al., Genome sequence of the button mushroom Agaricus bisporus reveals mechanisms governing adaptation to a humic-rich ecological niche. Proc Natl Acad Sci U S A (2012) 109: 17501-17506.

[15] L.M. Larraya, G. Pérez, E. Ritter, A.G. Pisabarro, L. Ramírez, Genetic linkage map of the edible basidiomycete Pleurotus ostreatus. Appl Environ Microbiol (2000) 66: 5290-5300.

[16] A. Jedinak, S. Dudhgaonkar, Q. Wu, J. Simon, D. Sliva, Anti-inflammatory activity of edible oyster mushroom is mediated through the inhibition of NF-אB and AP-1 signaling. Nutr J (2011) 10: 52.

[17] A. Jedinak, D. Sliva, Pleurotus ostreatus inhibits proliferation of human breast and colon cancer 
cells through p53-dependent as well as p53-independent pathway. Int J Oncol (2008) 33: 1307-1313.

[18] I.V. Grigoriev, H. Nordberg, I. Shabalov, A. Aerts, M. Cantor, D. Goodstein, et al., The genome portal of the department of energy joint genome institute. Nucleic Acids Res (2012) 40: D26-D32.

[19] K.H. Ma, G.A. Lee, S.Y. Lee, J.G. Gwag, T.S. Kim, W.S. Kong, et al., Development and characterization of new microsatellite markers for the oyster mushroom (Pleurotus ostreatus). J Microbiol Biotechnol (2009) 19: 851-857.

[20] R. Riley, A.A. Salamov, D.W. Brown, L.G. Nagy, D. Floudas, B.W. Held, et al., Extensive sampling of basidiomycete genomes demonstrates inadequacy of the white-rot/brown-rot paradigm for wood decay fungi. Proc Natl Acad Sci U S A (2014) 111: 9923-9928.

[21] Y.C. Li, A.B. Korol, T. Fahima, E. Nevo, Microsatellites within genes: structure, function, and evolution. Mol Biol Evol (2004) 21: 991-1007.

[22] P. Michalak, Coexpression, coregulation, and cofunctionality of neighboring genes in eukaryotic genomes. Genomics (2008) 91: 243.

[23] M.A. Harris, J.I. Deegan, J. Lomax, M. Ashburner, S. Tweedie, S. Carbon, et al., The gene ontology project in 2008. Nucleic Acids Res (2008) 36: D440-D444.

[24] A. Conesa, S. Götz, J.M. García-Gómez, J. Terol, M. Talón, M. Robles, Blast2GO: a universal tool for annotation, visualization and analysis in functional genomics research. Bioinformatics (2005) 21: $3674-3676$.

[25] N. Blüthgen, K. Brand, B. Cajavec, M. Swat, H. Herzel, D. Beule, Biological profiling of gene groups utilizing Gene Ontology. Genome Inform (2005) 16: 106-115.

[26] A. Goffeau, B. Barrell, H. Bussey, R. Davis, B. Dujon, H. Feldmann et al., Life with 6000 genes. Science (1996) 274: 546-567. 
[27] V. Wood, R. Gwilliam, M.A. Rajandream, M. Lyne, R. Lyne, A. Stewart, et al., The genome sequence of Schizosaccharomyces pombe. Nature (2002) 415: 871-880.

[28] R.A. Dean, N.J. Talbot, D.J. Ebbole, M.L. Farman, T.K. Mitchell, M.J. Orbach, et al., The genome sequence of the rice blast fungus Magnaporthe grisea. Nature (2005) 434: 980-986.

[29] R.A. Ohm, J.F. De Jong, L.G. Lugones, A. Aerts, E. Kothe, J.E. Stajich, et al., Genome sequence of the model mushroom Schizophyllum commune. Nat Biotechnol (2010) 28: 957-963.

[30] J.E. Stajich, S.K. Wilke, D. Ahrén, C.H. Au, B.W. Birren, M. Borodovsky, et al., Insights into evolution of multicellular fungi from the assembled chromosomes of the mushroom Coprinopsis cinerea (Coprinus cinereus). Proc Natl Acad Sci U S A (2010) 107: 11889-11894.

[31] F. Martin, A. Aerts, D. Ahrén, A. Brun, E. Danchin, F. Duchaussoy, et al., The genome of Laccaria bicolor provides insights into mycorrhizal symbiosis. Nature (2008) 452: 88-92.

[32] E. Morin, A. Kohler, A.R. Baker, M. Foulongne-Oriol, V. Lombard, L.G. Nagye, et al., Genome sequence of the button mushroom Agaricus bisporus reveals mechanisms governing adaptation to a humic-rich ecological niche. Proc Natl Acad Sci U S A (2012) 109: 17501-17506.

[33] R. Riley, W. Robert (2011). Phylogeny and comparative genome analysis of a Basidiomycete fungi. Lawrence Berkeley National Laboratory: Lawrence Berkeley National Laboratory. LBNL Paper LBNL-4662E-Poster. Retrieved from: http://www.escholarship.org/uc/item/0066t273.

[34] J. Ye, L. Fang, H. Zheng, Y. Zhang, J. Chen, Z. Zhang, et al., WEGO: a web tool for plotting GO annotations. Nucleic Acids Res (2006) 34: W293-W297.

[35] E.N. Trifonov, The tuning function of tandemly repeating sequences: a molecular device for fast adaptation. Evolutionary Theory and Processes: Modern Horizons, Kluwer Academic Publishers (2004): 115-138. 
432

433

434

435

436

437

438

439

440

441

442

443

444

445

446

447

448

449

450

451

452

453

[36] M. Rizki, Y. Tamai, Effects of different nitrogen rich substrates and their combination to the yield performance of oyster mushroom (Pleurotus ostreatus). World J Microbiol Biotechnol (2011) 27: 1695-1702.

[37] C. Sánchez, Cultivation of Pleurotus ostreatus and other edible mushrooms. Appl Microbiol Biotechnol (2010) 85: 1321-1337.

[38] T.M. Salame, D. Knop, D. Levinson, S.J. Mabjeesh, O. Yarden, Y. Hadar, Inactivation of a Pleurotus ostreatus versatile peroxidase-encoding gene (mnp2) results in reduced lignin degradation. Environ Microbiol (2014) 16: 265-277.

[39] E. Fernández-Fueyo, R. Castanera, F.J. Ruiz-Dueñas, M.F. López-Lucendo, L. Ramírez, A.G. Pisabarro, et al., Ligninolytic peroxidase gene expression by Pleurotus ostreatus: differential regulation in lignocellulose medium and effect of temperature and pH. Fungal Genet Biol (2014) 72: $150-161$.

[40] T.M. Salame, D. Knop, D. Levinson, S.J. Mabjeesh, O. Yarden, Y. Hadar, Release of Pleurotus ostreatus versatile-peroxidase from $\mathrm{Mn}^{2+}$ repression enhances anthropogenic and natural substrate degradation. PloS One (2012) 7: e52446.

[41] R. Castanera, G. Pérez, A. Omarini, M. Alfaro, A.G. Pisabarro, V. Faraco, et al., Transcriptional and enzymatic profiling of Pleurotus ostreatus laccase genes in submerged and solid-state fermentation cultures. Applied Environ Microbiol (2012) 78: 4037-4045.

[42] L. Ramírez, J.A. Oguiza, G. Pérez, J.L. Lavín, A. Omarini, F. Santoyo, et al., Genomics and transcriptomics characterization of genes expressed during postharvest at $4^{\circ} \mathrm{C}$ by the edible basidiomycete Pleurotus ostreatus. International Microbiology (2011) 14: 111-120.

[43] M. Mar-Alba, M.F. Santibánez-Koref, J.M. Hancock, Amino acid reiterations in yeast are 

process. J Mol Evol (1999) 49: 789-797. Microbiol (1970) 24: 501-524.

[45] D.H. Baker, G.L. Czarnecki-Maulden, Pharmacologic role of cysteine in ameliorating or exacerbating mineral toxicities. The Journal of Nutrition (1987) 117: 1003-1010. 125-130.

[47] M.V. Katti, R. Sami-Subbu, P.K. Ranjekar, V.S. Gupta, Amino acid repeat patterns in protein 


\section{Figure captions}

468

469

470

471

472

473

474

475

476

477

478

479

480

481

482

483

484

485

486

487

Figure 1: (a) Comparison of six repeat patterns of SSRs among nine fungi organisms.

The vertical axis is the number of SSRs per Mbp of genomic sequence. (b) Hierarchical clustering of the SSR distribution pattern. Five agaricomycotina fungi have a more similar SSR distribution than other species. The $\mathrm{x}$-axis is the similarity between each pair of species.

Figure 2: The SSR (red) and exon (blue) distribution throughout the PC15 genome. They have the complement curves in general. The unit length to compute the SSR and exon density is 50000bp. The correlation coefficient between SSR and exon density is -0.29 , with p-value $4.98 \times 10^{-15}$.

Figure 3: (a) The distribution pattern of SSRs for every scaffold of the PC15 genome, the z-axis is the number of SSRs. (b) The number of SSRs located in exons or non-coding regions.

Figure 4: The GO directed acyclic graphs (DAG) for significantly enriched functions of the SSR-containing gene set. There are three types of DAG: molecular function, biological process and cellular component. The red node represents that the GO function is over-represented in the SSR-containing gene set, while the green node represents that the GO function is under-represented in the SSR-containing gene set. The FDR cutoff of the Fisher's Exact Test is 0.05 .

Figure 5: (a) The heat map of the proportions of RAAs for seven fungi. (b) The hierarchical clustering result for the heat map. (c) The box plot of the proportions of RAAs in selected species. 


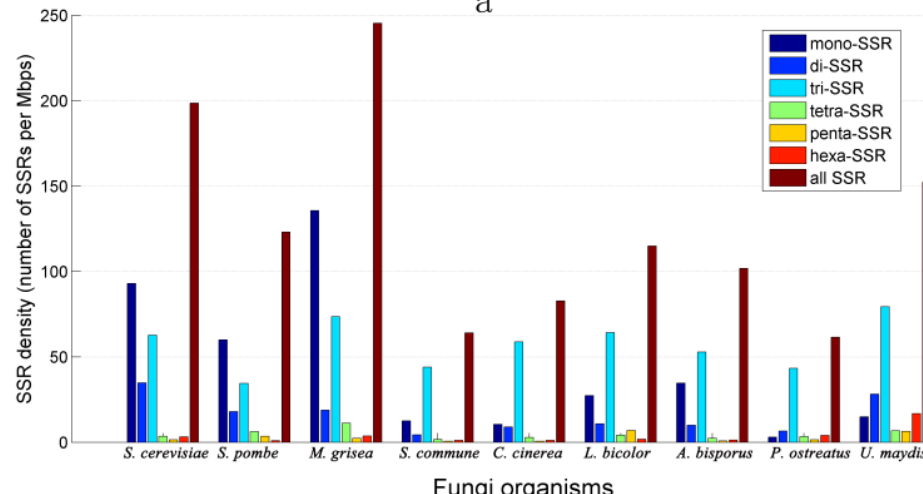

b

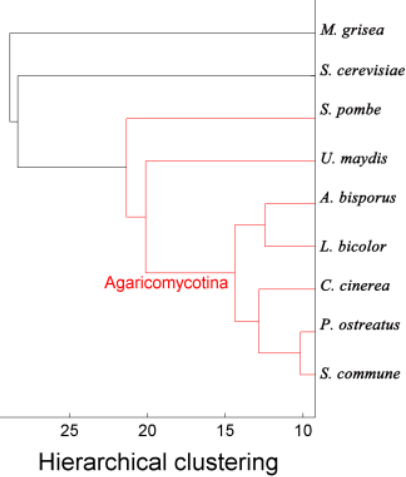

$490 \quad$ Figure 1 
491

492

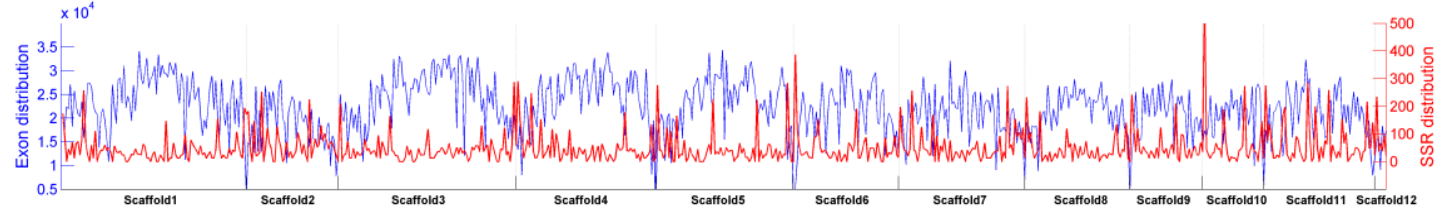

Figure 2 

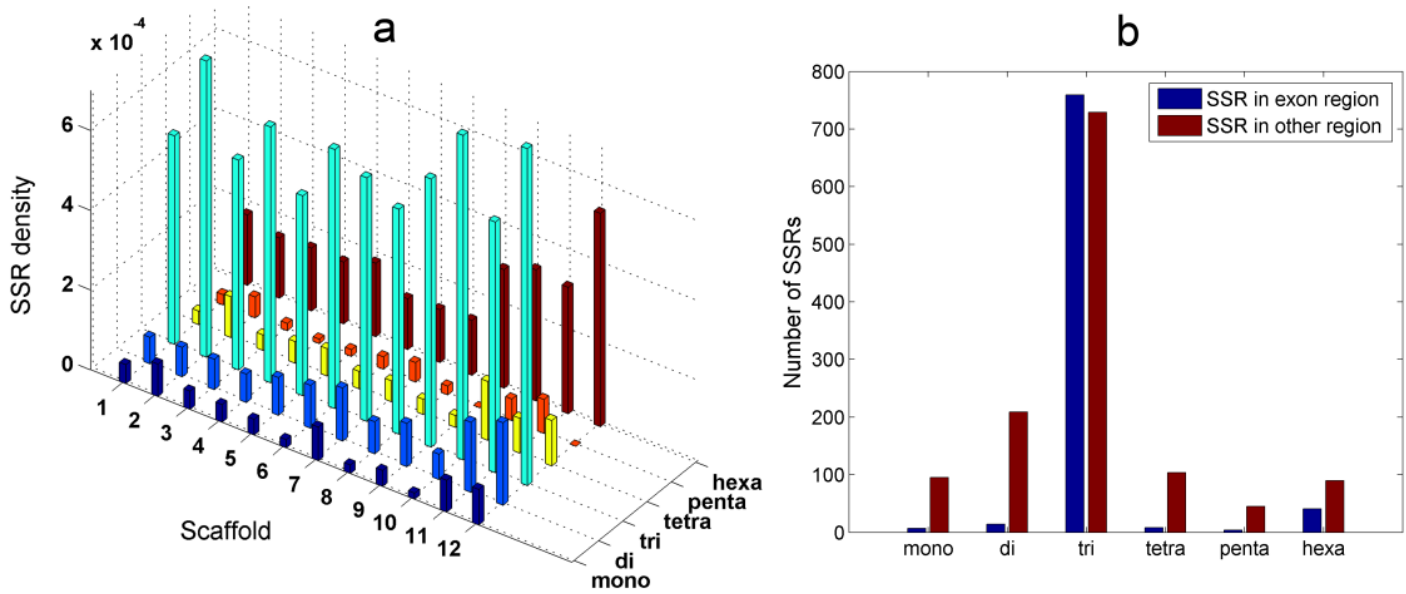

Figure 3 


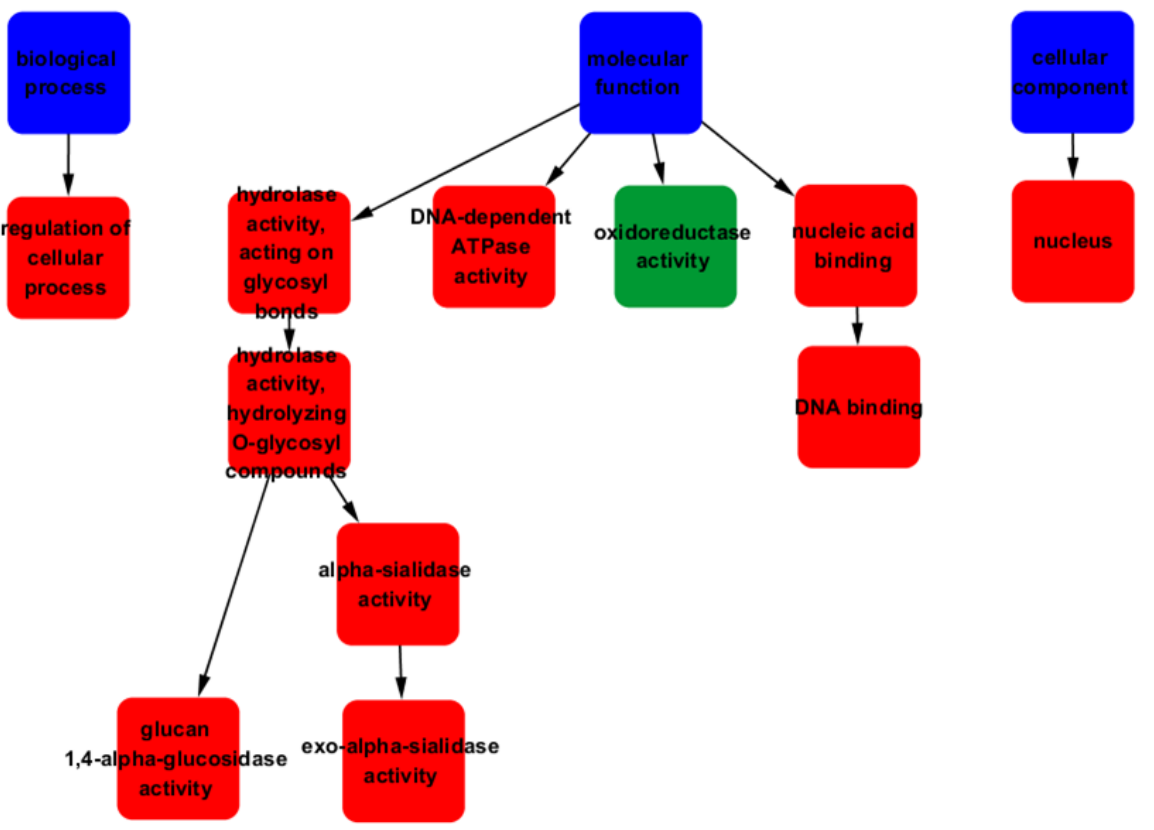

495

$496 \quad$ Figure 4 
(a) Proportions of repeat amino acids

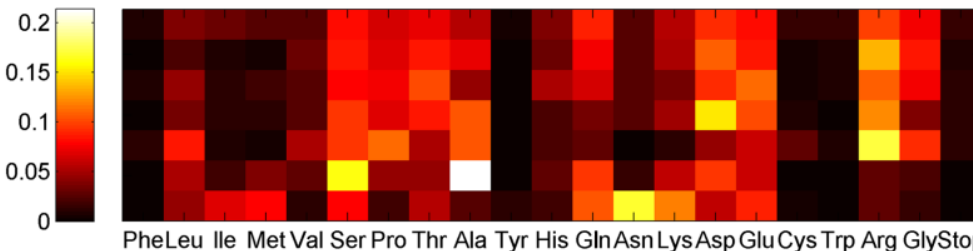

PheLeu Ile Met Val Ser Pro Thr Ala Tyr His GInAsn Lys Asp Glu Cys Trp Arg GlyStop

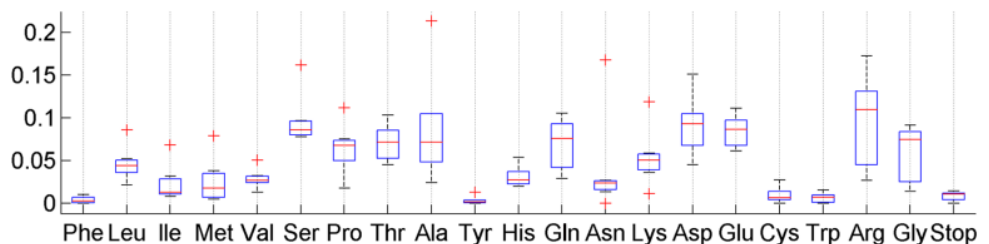

Phe Leu Ile Met Val Ser Pro Thr Ala Tyr His Gln Asn Lys Asp Glu Cys Trp Arg Gly Stop

(c) Boxplot for proportions of repeat amino acids (b) Hierarchical clustering

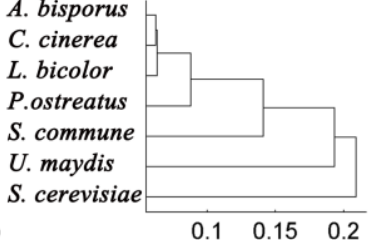

Figure 5 


\section{Table captions}

500 Table 1: The significant GO functions ( $\mathrm{p}$-value<0.01) for the 28 genes in regions 501 with abundant SSRs. GO-ID represents the unique term in Gene Ontology; Term is 502 the function name of the ID; Category is the three domains classified by GO: $\mathrm{C}$ is 503 cellular component, $\mathrm{F}$ is molecular function and $\mathrm{P}$ is biological process; $\mathrm{P}-\mathrm{V}$ alue is 504 computed by the Fisher's Exact Test.

505 Table 2: Details of the amino acids translated by trinucleotide SSRs in exons. 
Table 1: The significant GO functions ( $\mathrm{p}$-value $<0.01$ ) for the 28 genes in regions with abundant SSRs. GO-ID represents the unique term in Gene Ontology; Term is the function name of the ID; Category is the three domains classified by GO: $\mathrm{C}$ is cellular component, $\mathrm{F}$ is molecular function and $\mathrm{P}$ is biological process; $\mathrm{P}-\mathrm{V}$ alue is computed by the Fisher's Exact Test.

\begin{tabular}{|l|l|l|l|}
\hline GO-ID & Term & Category & P-Value \\
\hline GO:0006952 & defense response & P & $2.73 \mathrm{E}-03$ \\
\hline GO:0008766 & $\begin{array}{l}\text { UDP-N-acetylmuramoylalanyl-D-glutamyl-2,6-diaminopimelate-D- } \\
\text { alanyl-D-alanine ligase activity }\end{array}$ & F & $2.78 \mathrm{E}-03$ \\
\hline GO:0043773 & coenzyme F420-0 gamma-glutamyl ligase activity & F & $2.78 \mathrm{E}-03$ \\
\hline GO:0043774 & coenzyme F420-2 alpha-glutamyl ligase activity & F & $2.78 \mathrm{E}-03$ \\
\hline GO:0018169 & ribosomal S6-glutamic acid ligase activity & F & $2.78 \mathrm{E}-03$ \\
\hline GO:0070739 & protein-glutamic acid ligase activity & F & $2.78 \mathrm{E}-03$ \\
\hline GO:0008237 & metallopeptidase activity & F & $2.88 \mathrm{E}-03$ \\
\hline GO:0008451 & X-Pro aminopeptidase activity & F & $5.46 \mathrm{E}-03$ \\
\hline GO:0016881 & acid-amino acid ligase activity & F & $8.33 \mathrm{E}-03$ \\
\hline
\end{tabular}


Table 2: Details of the amino acids translated by trinucleotide SSRs in exons.

\begin{tabular}{|l|l|r|r|r|}
\hline Triplet & Amino acid & Repeat number & Molecular weight (g/mol) & Hydrophil \\
\hline 'gat/gac' & Aspartate(Asp) & 560 & 133.1 & 2.5 \\
\hline 'cgt/cgc/cga/cgg/aga/agg' & Arginine(Arg) & 455 & 174.2 & 3 \\
\hline 'gct/gcc/gca/gcg' & Alanine(Ala) & 389 & 89.1 & -0.5 \\
\hline 'gaa/gag' & Glutamate(Glu) & 374 & 147.1 & 2.5 \\
\hline 'tct/tcc/tca/tcg/agt/agc' & Serine(Ser) & 359 & 105.1 & 0.3 \\
\hline 'act/acc/aca/acg' & Threonine(Thr) & 310 & 119.1 & -0.4 \\
\hline 'cct/ccc/cca/ccg' & Proline(Pro) & 256 & 115.1 & -1.4 \\
\hline 'aaa/aag' & Lysine(Lys) & 179 & 146.2 & 3 \\
\hline 'ggt/ggc/gga/ggg' & Glycine(Gly) & 147 & 75.1 & 0 \\
\hline 'tta/ttg/ctt/ctc/cta/ctg' & Leucine(Leu) & 132 & 131.2 & -1.8 \\
\hline 'caa/cag' & Glutamine(Gln) & 128 & 146.2 & 0.2 \\
\hline 'gtt/gtc/gta/gtg' & Valine(Val) & 90 & 117.1 & -1.5 \\
\hline 'aat/aac' & Asparagine(Asn) & 88 & 132.1 & 0.2 \\
\hline 'cat/cac' & Histidine(His) & 84 & 155.2 & -0.5 \\
\hline 'att/atc/ata' & Isoleucine(Ile) & 45 & 131.2 & -1.8 \\
\hline 'taa/tag/tga' & Stop codon & 39 & & \\
\hline 'atg' & Methionine(Met) & 38 & 149.2 & -1.3 \\
\hline 'tgt/tgc' & Cysteine(Cys) & 26 & 121.2 & -1 \\
\hline 'tgg' & Tryptophan(Trp) & 9 & 204.2 & -3.4 \\
\hline 'ttt/ttc' & Phenylalanine(Phe) & 165.2 & -2.5 \\
\hline 'tat/tac' & Tyrosine(Tyr) & 4 & 181.2 & -2.3 \\
\hline & & 0 & & \\
\hline
\end{tabular}

